\title{
Cranial Ultrasound in Preterm Neonates: Screening of at Risk Infants for Long Term Neurodevelopmental Impairments
}

\author{
Mirza Md. Ziaul Islam ${ }^{1}$, Md. Atiqul Islam², M Monir Hossain ${ }^{3}$, Naila Zaman Khan ${ }^{4}$ \\ Received: April 27, 2015 Accepted: December 14, 2015 \\ doi: http://dx.doi.og/10.3329/jemc.v6i1.26379
}

\begin{abstract}
Background: Preterm birth is associated with variable degree of brain injury and adverse neurodevelopmental outcomes. Therefore, screening of these infants is required to assess further neurodevelopmental abnormalities. Cranial ultrasound finding has been a predictor of outcome in this population. Objective: The present study was carried out to find cranial ultrasound abnormalities in preterm neonates to aid in screening at risk infants for long term neurodevelopmenal impairment. Materials and Methods: This observational study was conducted in Dhaka Shishu (Children) Hospital from October 2011 to March 2012. Total 103 preterm neonates who met the inclusion criteria were enrolled in the study. The neonates underwent cranial ultrasound (cUS) scan just after admission and before discharge for screening at risk infants. Results: Total 103 neonates were enrolled with mean age of 7.67 days in neonates with normal cUS findings and 7.23 days in neonates with abnormal cUS findings. Mean gestational age was 31.94 weeks in neonates with normal cUS and 31.85 weeks in neonates with abnormal cUS findings with mean weight 1.83 and $1.81 \mathrm{~kg}$ respectively. Among the neonates, 64 (62.1\%) were males and 39 (37.9\%) were females. Before discharge, out of 103 preterm neonates, 56 (54.3\%) had normal cUS and 47 (45.7\%) had abnormal cUS findings of which 22 (21.4\%) had cerebral edema, 17 (16.5\%) IVH and 8 (7.8\%) had ventricular dilatation. Conclusion: Routine cranial ultrasound scanning of preterm neonates may aid in identifying at risk infants for long term neurodevelopmental impairments.
\end{abstract}

Key words: Preterm; Neonates; Cranial ultrasound; Neurodevelopment

J Enam Med Col 2016; 6(1): 28-32

\section{Introduction}

Premature birth is not normal. Being born prematurely is not a normal event despite its routine nature. ${ }^{1}$ Neonates born prematurely are at risk of brain injury. Although advances in neonatal care have greatly improved the survival and outcome of these vulnerable patients, brain injury still remains a major concern. Early diagnosis is important for prognostication, optimal treatment, and neurological outcome. ${ }^{2}$

The fetal or preterm infant brain is vulnerable to both hemorrhagic and ischemic injury during the late second and early third trimester. ${ }^{3}$ This is due to vascular, cellular and anatomical features of the developing brain, and the tendency for preterm infants to experience periods of physiological instability at a time when they have limited cerebral circulatory autoregulation. ${ }^{4}$ Even premature infants who have relatively uncomplicated neonatal causes are at substantial risk for developmental delays in cognition and motor skills. ${ }^{5}$ In summary, children born prematurely most often exhibit a combination of defects in cognitive, motor and behavioral performance and in interrelated functions.

1. Assistant Professor, Department of Infectious Diseases and Community Pediatrics, Dhaka Shishu Hospital, Dhaka

2. Registrar, Department of Infectious Diseases and Community Pediatrics, Dhaka Shishu Hospital, Dhaka

3. Professor, Department of Critical Care Unit, Dhaka Shishu Hospital, Dhaka

4. Professor, Department of Pediatric Neuroscience, Dhaka Shishu Hospital, Dhaka

Correspondence Mirza Md. Ziaul Islam, Email: mirzamd.ziaulislam@yahoo.com 
Despite being mild in degree individually, in combination, such deficits often lead to school difficulties, with a need for remedial assistance or speech education. ${ }^{6}$

An estimated $20 \%$ infants are born prematurely in Bangladesh, and 30\% have low birth weight (LBW) $(<2500 \mathrm{~g}) .^{7}$ With a total population of $>146$ million people including $>20$ million children $<5$ years of age ${ }^{8}$, large unrecognized populations may be at risk for neurodevelopmental morbidity, particularly considering that $85 \%$ of deliveries occur at home, often with no skilled care; only $7 \%$ births are ever registered and primary health care services do not include screening for the developmentally delayed child. ${ }^{9}$

Methodical issues remain regarding how to identify younger children with impairments and disabilities. Yet, there is awareness among parents of the presence of developmental problems early in the child's life. Recognizing impairments earlier might have improved their chances of catch-up development. ${ }^{10}$ Recent reviews of early intervention studies in high risk populations have demonstrated the potential to improve long term development in children across low and high income countries. ${ }^{11}$

Two main imaging techniques have been used for prediction of neurodevelopmental outcome in the neonatal period: cranial ultrasound (cUS) and MRI. ${ }^{6}$ The major benefits of testing are to direct families of preterm infants toward the most appropriate follow-up facilities to promote early diagnosis and intervention for chronic neurodevelopmental sequel of hemorrhagic or ischemic brain injury and to faster ongoing research activities that are aimed at ensuring the best possible outcomes for all infants. The major advantages of cUS are: it is relatively inexpensive and safe compared to other neuroimaging techniques, can be performed bedside with little manipulation of the infant. It can be repeated as often as necessary and thereby enables visualizations of ongoing brain maturation and the evaluation of brain lesion in addition to assessing the timing of brain damage. ${ }^{12}$ When cUS is used repeatedly, its sensitivity and specificity can be very high. ${ }^{13}$

Since the late 1970s, cUS examinations have been performed on preterm infants to provide information about perinatal brain injury for the prediction of longterm outcomes. ${ }^{14}$ Classic studies by Pape et $\mathrm{al}^{15}$ and
Slovis \& Kuhn ${ }^{16}$ reported on the use of real-time linear array and sector scanning to detect intraventricular and parenchymal hemorrhage, ventricular enlargement and other abnormalities. Major abnormalities on cUS predict the development of cerebral palsy (CP), neuromotor delay and cognitive outcome. ${ }^{17}$

In cranial ultrasound, images are interpreted normal if there is no hemorrhage, no evidence of dilatation. Some studies showed that a normal ultrasound scan provides considerable confidence that an infant will have normal neurodevelopment. ${ }^{18}$ The best predictor for outcome was a simple cranial ultrasound classification according to the presence or absence of ventriculomegaly and intraparenchymal damage of any cause. Infants with normal neonatal cerebral scan or abnormal scan without ventriculomegaly almost had a normal neurological outcome. In infants with cerebral lesions with ventriculomegaly, the incidence of normal neurological outcome decreased to less than $50 \%$. Intraparenchymal damage was associated with cerebral palsy as well as other (mental and sensory) handicaps in over $85 \%$ of the cases. ${ }^{19}$

The major clinical outcomes that are important to preterm infants and their families are survival and normal long term neurodevelopment. Regular follow-up assessments of children at risk of neurodevelopmental impairment may allow the early detection of problems and the provision of medical, social and educational support if required. It is recognized that support and active management of the at risk group of preterm infants may resolve or ameliorate potential later problems. The purpose of this study was to find cranial ultrasound findings in preterm neonates which may be utilized in counseling families about prognosis and the need for early intervention. This study may be of paramount importance in early identification and intervention to mitigate neurodevelopmental impairments in large populations that live in developing countries where professional expertise is sparse.

\section{Materials and Methods}

This observational study was carried out in Dhaka Shishu (Children) Hospital (DSH) from October 2011 to March 2012. Preterm neonates ( $<37$ weeks of gestational age) who were admitted in the neonatal unit of DSH were enrolled for the study. Neonates having perinatal asphyxia, congenital malformations especially of brain and spinal cord and neonatal conditions like 
respiratory distress syndrome (RDS), birth trauma were excluded from the study.

A structured questionnaire was used for recording all the information after taking written consent. All the preterm neonates underwent cranial ultrasound (cUS) imaging after admission and again before discharge for evaluation. Results are presented in tables. Statistical analysis was done by using software SPSS version 6.0 for Windows.

\section{Results}

In this study, 103 preterm neonates who were admitted in the neonatology unit of Dhaka Shishu (Children) Hospital during the study period from October 2011 to March 2012 were selected. All the preterm neonates underwent cranial ultrasound immediately after admission and before discharge. The findings from the data analysis are documented below.

Table I: Distribution of subjects according to age, gestational age and weight in both groups $(n=103)$

\begin{tabular}{|l|c|c|c|}
\hline Variables & $\begin{array}{c}\text { Normal cranial USG } \\
\text { findings }(\mathrm{n}=56)\end{array}$ & $\begin{array}{c}\text { Abnormal cranial } \\
\text { USG findings }(\mathrm{n}=47)\end{array}$ & P values \\
\hline $\begin{array}{l}\text { Age in days } \\
\text { (mean } \pm \text { SD) }\end{array}$ & $7.67 \pm 6.68$ & $7.23 \pm 5.75$ & 0.73 \\
\hline $\begin{array}{l}\text { Gestational age in } \\
\text { weeks (mean } \pm \text { SD) }\end{array}$ & $31.94 \pm 2.03$ & $31.85 \pm 1.91$ & 0.82 \\
\hline $\begin{array}{l}\text { Weight in kg } \\
\text { (mean } \pm \text { SD) }\end{array}$ & $1.83 \pm 0.20$ & $1.81 \pm 0.17$ & 0.54 \\
\hline
\end{tabular}

Among the 103 preterm infants $62.1 \%$ (64 infants) were male and $37.9 \%$ (39 infants) were female. Table II shows cranial ultrasound (cUS) findings of the neonates immediately after admission. Table III shows cranial ultrasound (cUS) findings of the neonates $(n=103)$ before discharge.

Table II: Cranial ultrasound (cUS) findings of the neonates immediately after admission $(n=103)$

\begin{tabular}{|l|c|c|}
\hline cUS findings & Number & Percentage \\
\hline Normal & 59 & 57.2 \\
\hline Abnormal & 44 & 42.8 \\
\hline Cerebral edema & 21 & 20.4 \\
\hline IVH & 15 & 14.6 \\
\hline Ventricular dilatation & 8 & 7.8 \\
\hline
\end{tabular}

IVH, intraventricular hemorrhage
Table III: Cranial ultrasound (cUS) findings of the neonates $(n=103)$ before discharge

\begin{tabular}{|l|c|c|}
\hline cUS findings & Number & Percentage \\
\hline Normal & 56 & 54.3 \\
\hline Abnormal & 47 & 45.7 \\
\hline Cerebral edema & 22 & 21.4 \\
\hline IVH & 17 & 16.5 \\
\hline Ventricular dilatation & 8 & 7.8 \\
\hline
\end{tabular}

IVH, intraventricular hemorrhage

The cranial ultrasound (cUS) findings of neonates immediately after admission and before discharge are shown in the algorithm below. The final cUS findings were: normal $56(54.3 \%)$, cerebral edema $22(21.4 \%)$, IVH 17 (16.5\%) and ventricular dilatation 8 (7.8\%).

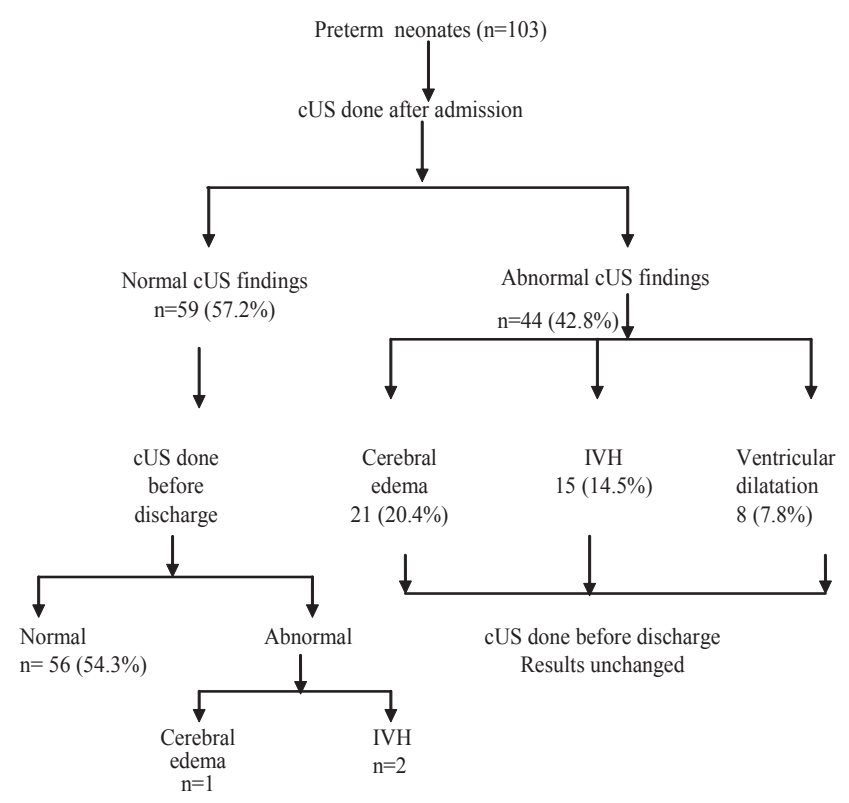

\section{Discussion}

Preterm birth is associated with variable degree of brain injury and adverse outcomes. Prediction of outcome after preterm birth is critical but remains difficult, particularly in the early postnatal period. The ability to predict outcome improves parental counseling and selection of infants for early therapeutic strategies aiming at preventing or ameliorating cerebral injury. ${ }^{21}$ Good prediction is usually achieved for major functional disabilities in early childhood. Cranial ultrasound (cUS) is a simple bedside tool which has very high sensitivity and specificity in predicting neurodevelopmental outcome when it is used repeatedly. ${ }^{13}$ 
In this study, the mean age (in days) of neonates with normal cUS finding was 7.67 and neonates with abnormal cUS finding was 7.23. Mean gestational age (in weeks) in neonates with normal cUS was 31.9 and with abnormal cUS was 31.85 and mean weight (in $\mathrm{kg}$ ) was 1.83 in neonates with normal cUS and 1.81 in neonates with abnormal cUS. Regarding sex distribution, $64(62.1 \%)$ neonates were male and 39 $(37.9 \%)$ were female. In one study ${ }^{22}$, gestational age (in weeks) was $29 \pm 0.9$ which is lower than that of this study. The explanation may be that prompt referral to large hospital with better management of preterm neonates in comparison to our health facilities may lead to survival of large number of very preterm neonates with lower birth weight.

Out of 103 preterm neonates, $56(54.3 \%)$ had normal cUS findings and 47 (45.63\%) had abnormal cUS findings. Among the abnormal cUS findings, 22 (21.4\%) had cerebral edema, $17(16.5 \%)$ had IVH and 8 (7.8\%) had ventricular dilatation. In one study ${ }^{17}$, cUS lesions were detected in the majority of infants (92\%) born $<32$ weeks of gestation. Another study ${ }^{21}$ found the incidence of IVH in preterm infants of less than $1500 \mathrm{~g}$ from $20-40 \%$. Another study ${ }^{23}$, conducted on 129 high risk preterm infants, showed that $51.63 \%$ had abnormal cUS findings of which $41.08 \%$ had IVH, $9.06 \%$ had ventricular dilatation.

Grazing \& Stainley $^{24}$ showed that ultrasound abnormalities were more strongly associated with delayed psychomotor development and the findings of ventriculomegaly and the diagnosis of periventricular hemorrhage were associated with more modest increased risk at 2 years of age.

Some other studies ${ }^{25-27}$ also found that ultrasound abnormalities are associated with a two to fourfold increase in the risk of low Bayley Scales of Infant Development (BSID)-II score and mental retardation. Another study ${ }^{28}$ revealed that major abnormalities on cUS predict the development of cerebral palsy (CP) and neuromotor delay at variable ages of follow-up.

Data from this study states that a substantial number of preterm neonates have abnormal cranial ultrasound findings and when repeated may identify the at risk infants. Long term follow-up and subsequent therapeutic intervention may ameliorate the severity of neurodevelopmental impairment. However, future studies in early months of life are recommended for routine screening of preterm neonates with cUS to detect risk infant. Finally, this study reveals that routine cranial ultrasound scan of preterm neonates may aid in identification of at risk infants for long term neurodevelopmental impairment.

\section{References}

1. Marlow M. Neurocognitive outcome after very preterm birth. Arch Dis Child Fetal Neonatal 2004; 89: 224-228.

2. van Wezel-Meijler G, Steggerda SJ, Leijser LM. Cranial ultrasonography in neonates: role and limitation. Acta Paediatr Scand 2009; 78: 142-153.

3. Volpe JJ. Brain injury in the premature infant. Neuropathology, clinical aspects, pathogenesis and prevention. Clin Perinatal 1997; 24: 567-587.

4. Pryds O. Control of cerebral circulation in the high risk neonate. Ann Neurol 1991; 30: 321-329.

5. Karen K, Mestal L, Jeremy D. Neurodevelopmental outcome of premature infants treated with inhaled nitric oxide. N Eng J Med 2005; 353: 23-32.

6. Beatrice L. Prediction of neurodevelopmental outcome after preterm birth, N Eng J Med 2009; 152: 42-47.

7. Hack U, Flannery DJ, Schulehte M. Saving newborn lives. State of the world's newborn, Washington DC: Save the Children Federation: 2001; 1-49.

8. Patra K. States of the world's children: childhood under threat. United Nations Children's Fund, NewYork 2005; 64-69.

9. Planning Wing, Ministry of Health and Family Welfare, Govt. of the People's Republic of Bangladesh. Conceptual Framework for Health, Nutrition and Population Sector Program (HNSPP), July 2003-June 2006; Dhaka, Bangladesh.

10. Khan NZ, Darmstadt GL. Neurodevelopmental outcome of preterm infants in Bangladesh. Pediatrics 2006; 118: 280-289.

11. Maulik PK, Darmstadtt GL. Community based interventions to optimize early childhood development in low resources settings. J Perinatol 2009; 29(8): 531-542.

12. Levene MI. Cranial ultrasound. British Medical Ultrasound Society (2006) in Medicine. Available at: www.bmus.org. Accessed May 2015.

13. De Vries LS, Groenendaal F. Neuroimaging in preterm infant. Ment Retard Dev Disabil Res Rev 2002; 8: 273-278.

14. Callahan TL, Hall JE, Ettner SL. The economic impact of multiple gestation pregnancies and the contribution of assisted-reproduction techniques to their incidence. $\mathrm{N}$ Eng $\mathrm{J}$ Med 1994; 33: 244-249. 
15. Pape KE, Blackwell RJ, Cusick G. Ultrasound detection of brain damage in preterm infants. Am J Neuroradiol 1997; 24: 1261-1264.

16. Slovis TL, Kuhn LR. Realtime sonography of the brain through the anterior fontanelle. Am J Roentgenol 1981; 136 : 277-286.

17. De Vries LS, Van Haastert ILC, Rademaker KJ. Ultrasound abnormalities preceding cerebral palsy in high risk preterm infants. J Pediatr 2004; 144: 815-820.

18. Nongena P, Ederies A, Azzopardi DV. Confidence in the prediction of neurodevelopmental outcome by cranial ultrasound and MRI in preterm infants. Arch Dis Child Fetal Neonatal, Ed 2010; 95: 388-390.

19. Weisglas-Kupereus N, Baerts W, Fetter WPE. Neonatal cerebral ultrasound, neonatal neurology and perinatal conditions as predictors of neurodevelopmental outcome in very low birth weight infants. Early Human Development, 1992; 31: 131-148.

20. Shaver DC, Bada HS, Korones SB. Early and late intraventricular hemorrhage: the role of obstetric factors. Obstet Gynecol; 1992; 80: 831-837.

21. Ferriero DM. Neonatal brain injury. N Eng J Med 2004; 35 : 1985-1995.

22. Van de Bor M, Ouden LD, Guit Gerard L. Value of cranial ultrasound \& MRI in predicting outcome in preterm infants. Pediatrics 1992; 90: 196.

23. Hankaran S, Bauer CR, Bain R. Prenatal and perinatal risk and protective factors for neonatal intracranial hemorrhage. Arc Pediatr Adolesc Med 150; 1996: 49-96.

24. Graziani LZ, Stailey PM. Cranial ultrasound and clinical studies in preterm infants. The Journal of Pediatrics 1985; 106: 269-276.

25. Bohr Br, Weight LL, Dusick AM. Neurodevelopmental and functional outcomes of extremely low birth weight infants in the National Institute of Child Health and Human Development. Neonatal Research network, 1993-94. Pediatrics 2000; 105: 1216-1226.

26. Whitaker AH, Feldman JF, Van Rossem. Neonatal cranial ultrasound abnormalities in low birth weight infants: relation to cognition outcomes at six years of age. Pediatrics 1998; 98: 719-729.

27. Van Bel F, den Ouden L, Van de Bor. Cerebral blood flow velocity during the first week of life of preterm infants and neurodevelopment at two years. Devmed Child Neurol 1989; 31: $320-328$.

28. Ancel PY, Livinee F, Larroque B. Cerebral palsy among preterm children in relation to gestational age and neonatal ultrasound abnormalities: the EP/PAGE cohort study. Pediatrics 2006; 117: 828-835. 